\title{
Catching a MeAsles OUtbreaK
}

$\mathbf{A}$ measles outbreak in the Central Sydney Health Area between February and April this year produced some interesting information about immunisation levels.

Two cases were reported to the Medical Officer of Health for Central and Southern Sydney by general practitioners on March 8. One was a six-year-old boy who attended an inner-city primary school. The other was a 12-year-old boy from an inner-city high school. The outbreak spread rapidly and, by March 26, 38 confirmed or suspected cases of measles had been identified.

In the first three weeks of March control measures included motivation of parents of cases and contacts to ensure that all unimmunised children were immunised. Immunisation sessions were held in all five schools where cases had been identified.

The proportion of pupils in each school who were immunised with measles/mumps/rubella vaccine varied from 4 per cent to 50 per cent. Initial poor response rates were probably due to an attempt to immunise only those children who had not been immunised previously. However, measles immunisation status was unknown for many children, so immunisation was offered to all children. Uptake rates improved after that decision was made.

During the third week of March, it became evident that there was a significant outbreak among Aboriginal children in the suburb of Redfern. An immunisation day was held in the local early childhood centre and the Aboriginal community was encouraged through doorknocks to take their childen for immunisation. During the door-knocks information was also sought on further cases of measles. On the immunisation day only seven children attended for measles immunisation.

On March 27, a case of measles was reported in a 16-year-old girl who attended an inner-city high school. No cases of measles had previously been identified in this school. Her 19-year-old cousin, who worked in a boarding school, had also contracted measles. It was not possible to undertake school-based immunisation before the Easter break and the school holidays. In an effort to control further spread of the outbreak the NSW Health Department issued a press release on March 28 which confirmed the presence of a measles outbreak and encouraged parents to ensure their children were immunised.

Over the Easter period several cases of measles were noted in a single street in the suburb of Glebe. With the cooperation of local general practitioners an immunisation stall was held in the street on Easter Sunday and Monday. Over the two days 95 children were immunised with measles/mumps/rubella vaccine.

After the holidays, all schools and day centres in the Central Sydney Area were contacted and asked to distribute a copy of a standard "dear parent" letter to each child. The letter described the measles outbreak, the possible complications of measles and encouraged all parents to ensure their children were immunised against measles. Schools and day care centres were asked to report all cases of measles to the Public Health
Unit. At the same time the local government councils of Sydney and South Sydney organised a letter drop to more than 30,000 homes in the inner-city, encouraging parents to immunise their children and providing information on council immunisation services.

The outbreak lasted about seven weeks. More than 80 per cent of cases occurred in the inner-city area. The index case was the 12-year-old whose measles was initially reported on March 8. The source of his infection is unknown.

Measles was confirmed in 46 children between 11 months and 19 years of age. A further nine cases of probable measles were identified. Several suspected cases were unable to be traced. Serological confirmation was obtained in six cases, including the index case. Of the confirmed cases, 23 were female and 23 male. No confirmed cases were admitted to hospital with a complication of measles.

Figure 1 shows the number of cases by age. Of the 46 confirmed cases 10 ( 22 per cent) occurred in the preschool age group (less than five years of age), 4 (9 per cent) occurred in children less than 15 months of age, and 2 (4 per cent) occurred in children less than 12 months of age.

Transmission occurred in a variety of ways - in schools, contact with neighbours and within families. There was no evidence of transmission occurring in day care centres; pre-school age children developed measles through contact with neighbours or family members.

Figure 2 shows the number of cases by date of onset of illness. The Medical Officer of Health was notified of the first cased on March 8- at the peak of the outbreak. Over the next three weeks control strategies included school-based immunisation programs and motivation of the families of cases and contacts. By March 28, when the NSW Health Department issued the press release, the incidence of measles was decreasing. Within one week of the Easter period, and the extensive media coverage at that time, the outbreak had ended.

Of the confirmed cases, 3 ( 7 per cent) had documented evidence of prior measles immunisation and 31 ( 67 per cent) had no history of measles immunisation. A history of measles immunisation was unable to be confirmed in 12 (26 per cent) cases. Measles immunisation histories which were obtained from parents of children in the "unconfirmed" group were, for the most part, very doubtful. It is likely that most, if not all, of these children were not immunised against measles.

If the efficacy of measles vaccine and the percentage of measles cases with a proven history of measles immunisation is known, then the percentage of the population which has been vaccinated against measles may be calculated (Anonymous, 1980). In the case of this outbreak only 7 per cent of cases had a confirmed history of measles immunisation. Assuming a vaccine efficacy of 95 per cent, the coverage rate for measles vaccination may be as low as 60 per cent in the affected areas. The spread of the outbreak among children who are mostly 


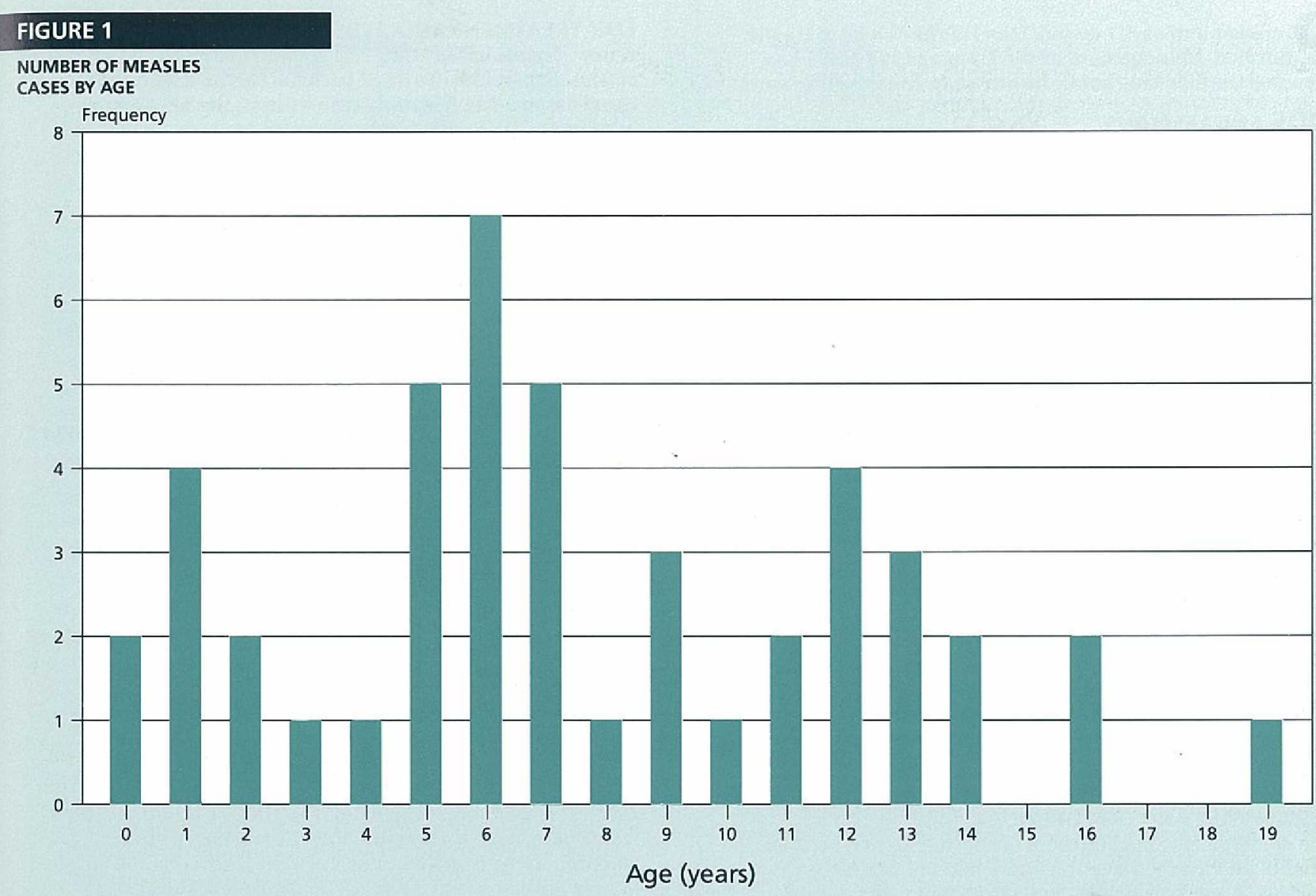

\section{FIGURE 2}

NUMBER OF MEASLES CASES

BY WEEK OF ONSET

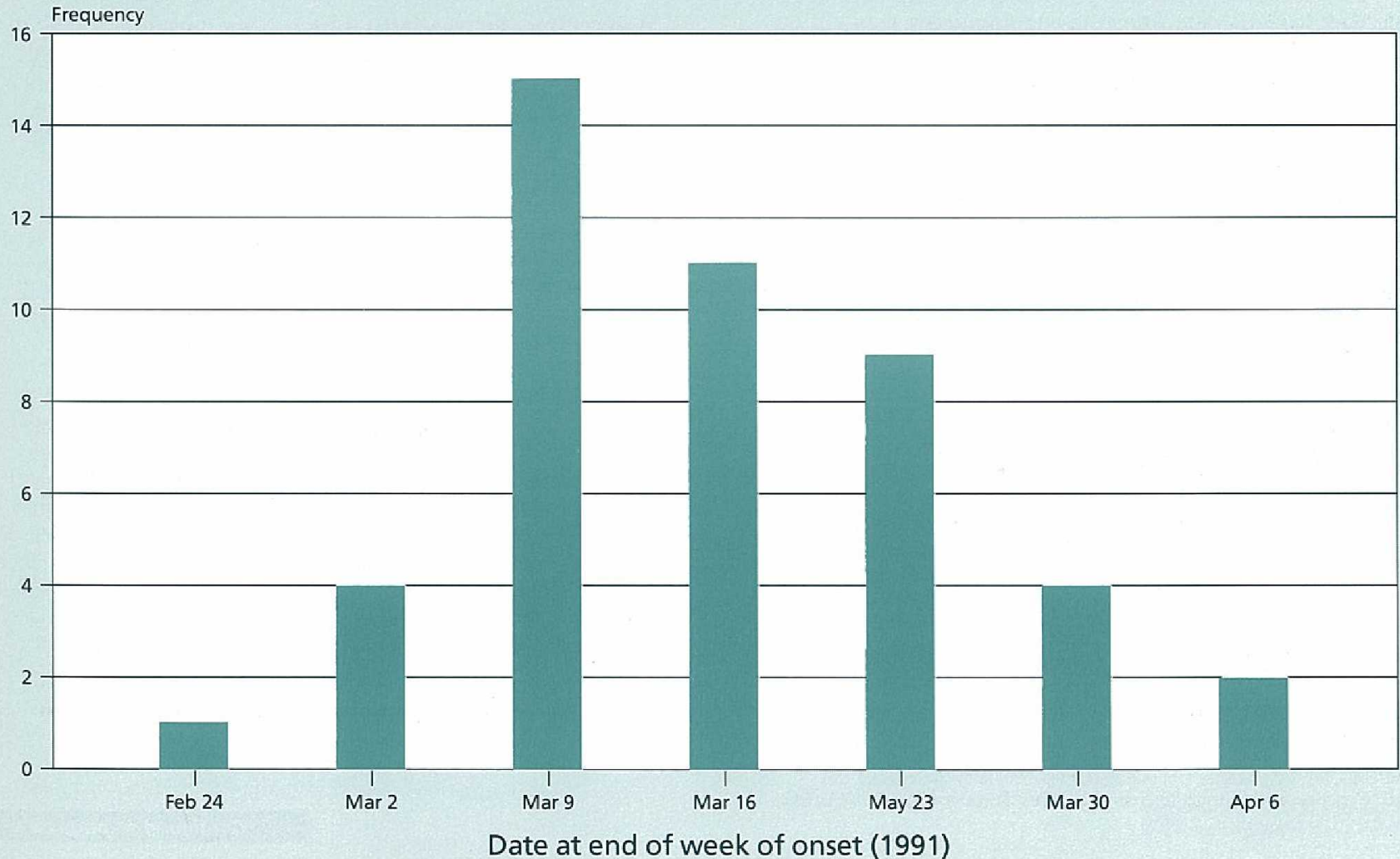




\section{Public Health Abstracts}

\section{Continued from page 68}

\section{UNEMPLOYMENT - HERE WE GO AGAIN}

Galbraith, the world's most literate economist, has said: "Let us remind ourselves what lies behind those numbers (on unemployment) - personal and family trauma, the loss of self-esteem, the tight-lipped fear about the future, the wonder as to whether there will be a job and income soon or ever again." These issues are crucial to health because all the radical solutions lie with economists and politicians, yet the evidence is that unemployment kills - particularly the middle-aged. There have been many studies in the past two decades which document that mortality is roughly a third higher in men seeking work than employed men. In 1990 a Finnish study found that mortality was 90 per cent higher among the unemployed than the employed after controlling for all the background variables. This Finnish study also showed that mortality increased with duration of unemployment. In all the studies, death rates are particularly high from suicide, accidents and violence and circulatory diseases.

Smith R, Unemployment: here we go again, Brit Med J 1991, 302, 606-607.

\section{FLUORIDATION - AN OLD CONTROVERSY}

Few public health activities engender more heat than the recurrent debate over fluoridation. In response to attacks led by scientists, the National Health and Medical Research Council's new working group to review the issues has issued an interim report. The basic conclusions are that fluoridation of water supplies does provide a good base for the reduction in dental decay in any community. The working group acknowledges that fluoridated toothpastes have also provided a major source of fluoride. Its basic recommendation is to encourage communities with reticulated water supplies, which are not fluoridated, to go ahead and fluoridate them.

If fluoride were removed from water supplies, the outcome would not be disastrous because of the high levels of fluoride and the widespread levels of fluoride-containing toothpastes and the increasing level of oral hygiene with these toothpastes.

Douglas RM, Fluoridation of Public Water Supplies, Med J Aust 1991, 154, 435-436.

\section{PREGNANCY AND THE OUTCOME OF MELANOMA}

The incidence of melanoma is rising and it appears

to be developing in twice as many women as men. Thus a large proportion of women who have had melanoma apparently successfully excised are still in their reproductive period. In view of the possibility of tumour sensitivity to hormones, women increasingly ask whether pregnancy after treatment alters the outlook, particularly with respect to melanomas diagnosed during a previous pregnancy. A large British study has shown that once the thickness of the melanoma tumour is controlled, the survival rate of pregnant women in whom melanoma was diagnosed and treated did not differ from that of other women. Therefore women with melanoma should be advised about pregnancy on the basis of thickness and site of tumour and evidence of vascular spread and not on hormonal status (this is a major change in practice in a very important and common field for Australia - JSL).

MacKie RM, Bufalino R, Morabito A, Lack of Effect of Pregnancy on Outcome of Melanoma, Lancet 1991, 337, 653-654

\section{Catching a Measles Outbreak}

\section{Continued from page 65}

unimmunised and who are resident in the inner-city area indicates a need to review childhood immunisation strategies in this area.

Delayed reporting of measles cases resulted in control measures not being instituted until the outbreak was well under way. The investigation of the outbreak showed that, of the two cases reported on March 8, one was a secondary contact of the index case. The index case was not reported until serological confirmation had been obtained - two weeks after the onset of illness. It is likely that many cases of measles could have been prevented if the doctor had notified the case on suspicion of measles rather than waiting for serological confirmation.

The control of a measles outbreak rests on the rapid immunisation of susceptible children. In this outbreak, measles immunisation was recommended for all unimmunised children over one year of age. Measles immunisation is not usually recommended for children less than six months of age because maternal antibody levels are usually sufficient to prevent infection. Measles immunisation may be given to children aged 6 to 12 months - but a second dose is recommended at 15 months of age. Alternatively, human immunoglobulin may be given to provide temporary protection. In this outbreak, the lack of any clear guidelines for protection of children aged 6 to 12 months caused some confusion among health care workers and the public. It is therefore important, in the event of a measles outbreak, that health care workers be provided with clear guidelines for this age group.

Lee Taylor

Public Health Officer

Central and Southern Sydney

Anonymous. Measles vaccine efficacy - United States. MMWR, 1980;29: $470-472$.

\section{PUBLIC HEALTH BULLETIN EDITORIAL STAFF}

The Bulletin's editorial advisory panel is as follows: Dr Sue Morey, Chief Health Officer, Department of Health; Professor Stephen Leeder, Professor of Community Medicine, University of Sydney; Professor Geoffrey Berry, Professor of Epidemiology \& Biostatistics, University of Sydney; Dr Christine Bennett, Associate Director, Service Development, Department of Health; Dr Michael Frommer, Epidemiologist, Epidemiology \& Health Services Evaluation Branch; Jane Hall, Director, NSW Centre for Health Economics, Research and Evaluation, Department of Community Medicine, Westmead Hospital; and Mr Michael Ward, Manager, Health Promotion Unit, Department of Health.

The editor is Dr George Rubin, Director, Epidemiology and Health Services Evaluation Branch, Department of Health, NSW.

Design and Production - Health Public Affairs Unit, Department of Health, NSW.

Please send your articles, news, comments or letters to Dr George Rubin - Locked Bag 961, North Sydney NSW 2059 or Fax (02) 3919232.

Suggestions for improving the content of the Bulletin are welcome. 